\title{
Patients with acute coronary syndromes express enhanced CD40 ligand/CD154 on platelets
}

C D Garlichs, S Eskafi, D Raaz, A Schmidt, J Ludwig, M Herrmann, L Klinghammer, W G Daniel, A Schmeisser

\begin{abstract}
Objective-To investigate whether CD40L/CD154 on platelets and soluble CD40L/CD154 may play a role in the inflammatory process of acute coronary syndromes.

Design and setting-Observational study in a university hospital.

Patients -15 patients with acute myocardial infarction, 25 patients with unstable angina, 15 patients with stable angina, and 12 controls.

Main outcome measures-CD40L/CD154 on platelets, P-selectin/CD62P on platelets, soluble CD40L/CD154 serum concentrations.

Results-Mean (SD) CD40L/CD154 expression on platelets was 6.2 (2.8) MFI (mean fluorescence intensity) in the infarct group, 11 (3.3) MFI in the unstable angina group ( $p<0.001 v$ infarction), $3.6(0.9) \mathrm{MFI}$ in the stable angina group ( $\mathrm{p}<0.01 v$ infarction; $\mathrm{p}<0.001 v$ unstable angina), and 3.2 (1.0) MFI in the controls ( $\mathrm{p}<0.01 v$ infarction; $\mathrm{p}<0.001 v$ unstable angina; NS $v$ stable angina). Soluble CD40L/CD154 concentration was $5.2(1.1) \mathrm{ng} / \mathrm{ml}$ in the infarct group, $4.2(0.7) \mathrm{ng} / \mathrm{ml}$ in the unstable angina group ( $\mathrm{p}<0.001 v$ infarction), $2.9(1.0) \mathrm{ng} / \mathrm{ml}$ in stable angina group ( $\mathrm{p}<0.001 \mathrm{v}$ infarction and unstable angina), and $3.0(0.5) \mathrm{ng} / \mathrm{ml}$ in the controls $(\mathrm{p}<0.001 v$ infarction and unstable angina; NS $v$ stable angina). At a six months follow up, there was lower expression of CD40L/CD154 on platelets in patients with unstable angina (12.3 (3.6) v 3.8 (1.2) MFI, p < 0.0001) and acute myocardial infarction (6.2 (2.8) v 3.5 (0.8) MFI, $\mathrm{p}<0.01)$ compared with their admission values six months earlier. Patients with unstable angina who needed redo coronary angioplasty (PTCA) or who had recurrence of angina were characterised by increased CD40L/CD154 expression on platelets compared with the remainder of the study group (recurrence of angina: 12.7 (3.2) v 9.7 (1.6) MFI, p < 0.05; re-do PTCA: 14.3 (4.2) $v 10.3$ (2.1) MFI, $\mathrm{p}<0.05)$.

Conclusions-Both CD40L/CD154 on platelets and soluble CD40L/CD154 are raised in patients with unstable angina and myocardial infarction. These findings suggest that CD40-CD40L/CD154 interactions may play a pathogenic role in triggering and propagation of acute coronary syndromes.

(Heart 2001;86:649-655)
\end{abstract}

Keywords: acute coronary syndromes; unstable angina, CD40L/CD154; platelets

Atherosclerosis is an inflammatory disease. ${ }^{1}$ Patients with coronary artery disease, particularly those with unstable angina, have raised serum concentrations of circulating proinflammatory cytokines and soluble adhesion molecules. ${ }^{23}$ Furthermore, extensive infiltration of blood derived macrophages and $\mathrm{T}$ cells into the vessel wall is an important feature of the active stages of atherosclerosis. ${ }^{145}$

Recently, several studies have suggested that CD40-CD40L/CD154 interactions, which are known to be essential in immune reactions and autoimmune diseases, ${ }^{6}$ are also important in atherosclerosis. $^{7-12}$ CD40 ligand (CD40L/ CD154) is a transmembrane protein structurally related to tumour necrosis factor $\alpha(\mathrm{TNF} \alpha)$ and was originally identified on stimulated $\mathrm{CD} 4{ }^{+} \mathrm{T}$ cells, ${ }^{13}$ and later on stimulated mast cells, basophils, and vascular cells such as smooth muscle cells. ${ }^{14}{ }^{15}$ The receptor CD40 is constitutively expressed on B cells, monocytes, macrophages, endothelial cells, and dendritic cells. ${ }^{16-18}$

Ligation of CD40 on atheroma associated cells causes activation of matrix metalloproteinases $^{19}$ and enhanced production of cellular adhesion molecules, proinflammatory cytokines, chemokines, ${ }^{20}$ and tissue factor, ${ }^{21} 22$ and thus leads to a progression of atherosclerosis, plaque destabilisation, and the formation of intravascular thrombus. The blockade of CD40-CD40L/ CD154 interactions by administration of a blocking antibody against CD40L/CD154 in mice deficient in low density lipoprotein receptor or ApoE has been shown to reduce atherosclerosis in both initial and advanced stages, and to induce a stable plaque phenotype..$^{7-10}$

CD40L/CD154 has recently been described on platelets. It can be expressed within seconds after activation of platelets and can induce an inflammatory reaction in endothelial cells. ${ }^{23} \mathrm{It}$ is well known that activation of platelets plays a crucial role in acute coronary syndromes ${ }^{24}$ - for example, compared with stable angina, patients with unstable angina have increased surface expression of $\mathrm{P}$-selectin/CD62P and activated GP IIb/IIIa complex (LIPS-1), representing a prothrombotic state. ${ }^{25}$

Despite of the proof of biologically functional CD40L/CD154 on platelets, up to now there has been little information about CD40L/CD154 on platelets in patients with stable or unstable angina, ${ }^{26}$ or in patients with acute myocardial infarction. To investigate a 
Table 1 Baseline characteristics of the study groups

\begin{tabular}{|c|c|c|c|c|}
\hline & $\begin{array}{l}\text { Stable AP } \\
(n=15)\end{array}$ & $\begin{array}{l}\text { Unstable AP } \\
(n=25)\end{array}$ & $A M I(n=15)$ & $p$ Value \\
\hline Age (years) & $66.3(8.4)$ & $67.9(10.5)$ & $63.5(11.8)$ & NS \\
\hline Sex,$M / F(\%)$ & $83.3 / 16.7$ & $62.5 / 37.5$ & $66.7 / 33.3$ & NS \\
\hline Smokers $(\%)$ & 39.1 & 41.7 & 41.7 & NS \\
\hline \multicolumn{5}{|l|}{ Drug treatment $(\%)$} \\
\hline Heparin (unfractionated) & 0 & 52 & 100 & \\
\hline Aspirin & 83.3 & 91.3 & 72.7 & \\
\hline$\beta$ Blocker & 75 & 82.6 & 63.6 & \\
\hline Calcium antagonist & 8.3 & 17.4 & 27.3 & \\
\hline Long acting nitrates & 66.7 & 61 & 30 & \\
\hline Infusion of glyceryl trinitrate & 0 & 8.7 & 0 & \\
\hline $\begin{array}{l}\text { HMG-CoA reductase } \\
\text { inhibitors }\end{array}$ & 50 & 56.5 & 60 & \\
\hline \multicolumn{5}{|c|}{ Number of affected coronary arteries (\%) } \\
\hline 1 & 36.4 & 22.7 & 36.4 & \\
\hline 2 & 45.4 & 45.5 & 54.5 & \\
\hline 3 & 18.2 & 31.8 & 9.1 & \\
\hline Total cholesterol $(\mathrm{mmol} / \mathrm{l})$ & $5.22(1.37)$ & $5.72(1.42)$ & $5.05(1.14)$ & NS \\
\hline LDL cholesterol $(\mathrm{mmol} / \mathrm{l})$ & $3.11(0.94)$ & $3.33(1.55)$ & $2.78(1.08)$ & NS \\
\hline HDL cholesterol $(\mathrm{mmol} / \mathrm{l})$ & $1.14(0.35)$ & $1.25(0.46)$ & $1.21(0.24)$ & NS \\
\hline Triglycerides $(\mathrm{mmol} / \mathrm{l})$ & $1.89(0.65)$ & $2.12(1.15)$ & $169.3(1.32)$ & NS \\
\hline $\mathrm{C}$ reactive protein $(\mathrm{mg} / \mathrm{l})$ & $0.25(0.1)$ & $5.74(2.2)^{\star}$ & $8.2(3.8)^{\star} \dagger$ & ${ }^{\star} \mathrm{p}<0.05$ ( $v$ control $) ; \uparrow \mathrm{NS}(v$ UAP $)$ \\
\hline Creatine kinase (U/l) & $36.6(25.2)$ & $52.1(34.0)$ & $289.1(88.4)^{\star}$ & ${ }^{\star} \mathrm{p}<0.001(v \mathrm{SAP} / \mathrm{UAP})$ \\
\hline CK-MB (\%) & NM & NM & $13.48(6.0)$ & \\
\hline Troponin I (ng/ml) & $0.17(0.1)$ & $1.01(0.3)^{\star}$ & $17.64(2.5)^{\star} \dagger$ & ${ }^{\star} \mathrm{p}<0.001(v \mathrm{SAP}) ;+\mathrm{p}<0.001(v \mathrm{UAP})$ \\
\hline Platelets $(10 / \mu \mathrm{l})$ & $232(77)$ & $247(68)$ & $275(116)$ & NS \\
\hline
\end{tabular}

Data are means (SD) or \%.

AMI, acute myocardial infarction; AP, angina pectoris; F, female; HDL, high density lipoprotein; $M$, male; NM, not measured.

possible involvement of CD40-CD40L/ CD154 interactions in the triggering of acute coronary events, we investigated whether patients with various types of coronary syndrome show significant differences in the expression of CD40L/CD154 on their platelets. Because raised serum concentrations of soluble CD40L/CD154 (sCD40L/CD154) have recently been reported in patients with unstable angina, ${ }^{27}$ we determined serum concentration of sCD40L/CD154 in patients with stable angina, unstable angina, and acute myocardial infarction. In addition to CD40L/CD154 on platelets, we analysed membrane bound $\mathrm{P}$-selectin/CD62P as a marker of platelet activation in all study groups.

\section{Methods}

PATIENTS AND CONTROLS

Between January 1999 and May 2000, details of all patients with angiographically proven coronary artery disease (stenosis of $>75 \%$ in at least one coronary vessel) were recorded and the patient population was derived from these (table 1). The study was approved by the institutional ethics committee for human subjects. Informed consent was obtained from all patients.

\section{Acute myocardial infarction}

Fifteen patients with acute myocardial infarction were selected. Inclusion criteria were: typical anginal pain lasting $>30$ minutes; ST segment elevation of $\geqslant 1 \mathrm{~mm}$ in two or more contiguous leads; and elevation of serum creatine kinase to $\geqslant 3$ times the upper limit of normal, with a concomitant rise in the MB isoenzyme. At least two of these three criteria had to be met.

Unstable angina

Twenty five consecutive patients fulfilling the criteria for unstable angina were selected. Unstable angina was defined as rest pain occurring within 48 hours without a recent myocardial infarct (Braunwald class IIIB). These patients had no evidence of "major" myocardial necrosis, as reflected by raised serum creatine kinase or creatine kinase $\mathrm{MB}$ isoenzyme, but there was minor myocardial injury detected by repeated measurements of troponin I (Braunwald class IIIB-T positive $v$ IIIB$\mathrm{T}_{\text {negativ }}$ ). ${ }^{28}$ Transient ST-T segment depression and $T$ wave inversion were often present in this group of patients.

Blood from patients with unstable angina or acute myocardial infarction was drawn immediately after their admission to the hospital.

Stable angina

In the same period, 15 sex and age matched patients with stable angina were selected among consecutive patients undergoing elective diagnostic coronary angiography or percutaneous transluminal coronary angioplasty (PTCA). All of these latter patients had long term (more than six months) stable effort angina and a positive exercise test. Drug treatment in this group often included aspirin and nitrates.

\section{Controls}

The control group consisted of 12 sex and age matched patients with atypical chest pain who underwent coronary angiography for exclusion of coronary artery disease.

\section{Exclusion criteria}

Exclusion criteria for all groups were: noncardiac diseases that might interfere with the findings (anaemia, infection, malignant disease, collagen diseases, hyperthyroidism); cardiac disease other than coronary artery disease, except for minor mitral regurgitation; overt right or left ventricular failure; and coronary artery bypass surgery, balloon angioplasty, and thrombolytic treatment within three months of the study. 
BLOOD SAMPLING PROTOCOL

Peripheral venous blood was drawn into blood collection tubes containing $106 \mathrm{mmol} / \mathrm{l}$ sodium citrate, immediately transferred to the laboratory, centrifuged at $190 \mathrm{~g}$ for 10 minutes at room temperature, and thereafter used for obtaining platelet-rich plasma (PRP, see below). A separate aliquot of blood without any additives was immediately immersed in melting ice and allowed to clot for one hour before centrifugation $\left(1500 \mathrm{~g}\right.$ at $4^{\circ} \mathrm{C}$ for 10 minutes). The serum supernatant was stored at $-80^{\circ} \mathrm{C}$ until analysis. Samples were thawed only once. The blood collection generally occurred before administration of drugs, except in the case of heparin and aspirin.

IMMUNOFLUORESCENCE

Platelet immunostaining was performed as previously described. ${ }^{29}$ Briefly, PRP was prepared after centrifugation with $190 \mathrm{~g}$ at room temperature for 10 minutes. The number of platelets was adjusted to $20000 / \mu 1$ using phosphate buffered saline. Aliquots of PRP were mixed 1:8 with fluorescein isothiocyanate (FITC) conjugated anti-CD62P (AK-4, IgG $\left.{ }_{1}\right)$ or phycoerythrin conjugated anti-CD40L (TRAP1, IgG ${ }_{1}$ ) and incubated for 30 minutes at room temperature. Thereafter cells were diluted with $1 \mathrm{ml}$ phosphate buffered saline and immediately analysed by flow cytometry. Five thousand cells were measured by flow cytometry (FACS Calibur, Heidelberg, Becton Dickinson) within six hours of sampling and analysed by CellQuest Software (Becton Dickinson). Platelets were identified with antiCD41a (HIP 8, IgG ${ }_{1}$, phycoerythrin, or FITC conjugated), which labelled all platelets. Platelets were gated according to staining for the platelet specific antigen, CD41a. The platelet population evaluated was found to be $\geqslant 98 \%$ positive for CD41a. FITC and phycoerythrin conjugated mouse $\operatorname{IgG}_{1}$ (MOPC-21) served as isotype controls. All antibodies were purchased from PharMingen (Hamburg, Germany).

EIA FOR SOLUBLE CD40L/CD154

Concentrations of sCD40L/CD154 were determined using a commercially available enzyme immunoassay (detection limit $95 \mathrm{pg} /$ ml; Bender MedSystems, Vienna, Austria) according to the manufacturer's instructions.

ANGIOGRAPHIC ANALYSIS

Atherosclerotic lesions were graded according to the American College of Cardiology/ American Heart Association classification of lesion type. ${ }^{30}$ Briefly, type $A$ lesions were defined as discrete lesions ( $<10 \mathrm{~mm}$ in length) with a smooth contour, little or no calcification, absence of thrombus, and less than total occlusion in areas not involving branching points. Type $B$ lesions were tubular lesions $(10-20 \mathrm{~mm}$ in length) with an irregular contour, moderate to heavy calcification, total occlusion $(<3$ months old), or low level thrombus formation in areas involving bifurcation. Type $C$ lesions were defined as diffuse lesions $(>2 \mathrm{~cm}$ in length) with excessive tortuosity of the proximal segment or total occlusion ( $>3$ months old). Severe cases of vein graft degeneration with friable lesions were also included among type C lesions.

TIMI flow grade (antegrade flow grade through stenotic/occluded arteries) was defined as previously described. ${ }^{31}$ Grade 0 was defined as complete absence of antegrade flow; grade I as presence of antegrade flow but with failure to fill the distal vessels; grade II as delayed filling of the distal vessels; and grade III as complete antegrade flow without delay.

\section{STATISTICAL ANALYSIS}

For each treatment the geometrical mean fluorescence intensity value (MFI) for the control stained population was subtracted from the MFI value of the positively stained sample. MFI are the geometric mean intensities and refer to values normalised to the log scale.

Explorative data analysis was performed to test the hypothesis that the distribution within the groups and subgroups was normal, to decide the applicability of parametric tests. Outliers were excluded. When comparing normally distributed variables in four groups of individuals, one way analysis of variance was followed by the Tukey post hoc test for significance. Subgroup comparisons of proportions were made by the $\chi^{2}$ test. The significance of differences between two subgroups was assessed by unpaired $t$ testing or by the non-parametric Wilcoxon-Mann-Whitney U test. For comparison within the same individual over time, the Wilcoxon matched pairs test was used.

Values are given as mean (SD). A probability value of $p<0.05$ was taken to indicate statistical significance.

\section{Results}

Table 1 summarises the baseline characteristics of the patients with stable angina, unstable angina, and acute myocardial infarction. All groups were comparable with respect to age, sex, risk factor profile, and platelet counts. In the serum analyses, patients with unstable angina and acute myocardial infarction had significantly higher concentrations of $\mathrm{C}$ reactive protein and troponin I than patients with stable angina or controls.

Patients with unstable angina and to a lesser extent those with acute myocardial infarction were found to have increased expression of CD40L/CD154 on platelets, as shown in fig 1. Patients with unstable angina had significantly higher concentrations of CD40L/CD154 on platelets than patients with stable angina (11 (3.3) v $3.6(0.9) \mathrm{MFI}$ ), acute myocardial infarction (6.2 (2.8) MFI), or controls (3.2 (1.0) MFI) (all $\mathrm{p}<0.001)$. CD40L/CD154 expression on platelets from patients with acute myocardial infarction, while significantly lower than in the unstable angina group, was increased over the stable angina and control groups (all $\mathrm{p}<0.01$ ). No significant differences in CD40L/ CD154 expression were detected between patients with stable angina and controls $(p=0.3)$.

As well as comparing the total intensity of CD40L/CD154 positive platelets, we also compared the percentage of platelets positive for expression of CD40L/CD154 in each test 


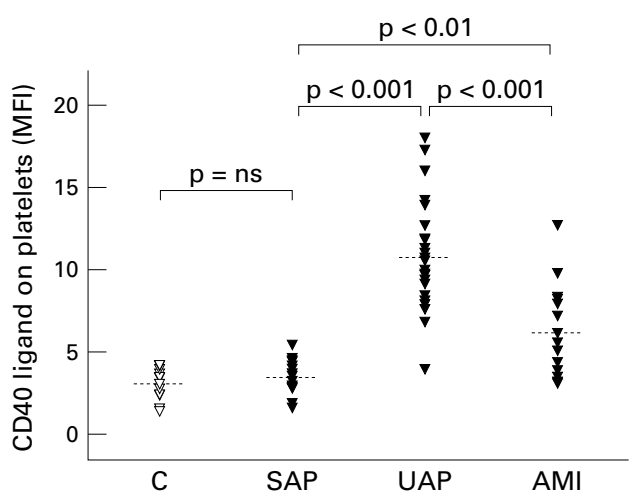

Figure 1 CD4OL/CD154 on platelets in 12 controls (C), 15 patients with stable (SAP), 25 patients with unstable angina (UAP), and 15 patients with acute myocardial infarction (AMI). Symbols represent individual measurements (mean fluorescence intensity ((MFI)), the dotted line the mean value of each group ( $n s$, non-significant).

subject; patients with unstable angina had a higher percentage $(12.3(3.1) \%)$ than patients with acute myocardial infarction $(7.2(2.3) \%)$, patients with stable angina $(3.6(1.2) \%)$, or controls (3.1 (1.0)\%). At a six months follow up, expression of CD40L/CD154 on platelets was reduced in patients with unstable angina (3.8 (1.2) v 12.3 (3.6) MFI; p < 0.001, fig 2) and acute myocardial infarction $(3.5(0.8) v$ 6.2 (2.8) MFI; p < 0.01, data not shown) compared with the values at the time of their admission six months earlier.

An analysis of the correlation among the variables showed no relation between the concentrations of CD40L/CD154 on platelets and the individual drugs taken by the patients, including those with known effects on platelet function (aspirin and heparin). In addition, no correlation was found between CD40L/ CD154 on platelets and sex, C reactive protein, lipids, or smoking status in patients with stable angina, unstable angina, or acute myocardial infarction (data not shown).

Because membrane bound CD40L/CD154 from $\mathrm{T}$ cells and platelets can be shed into the circulation, we analysed sCD40L/CD154 in all the study groups. As shown in fig 3, raised concentrations of sCD40L/CD154 were detected in patients with unstable angina and to a greater extent those with myocardial infarction

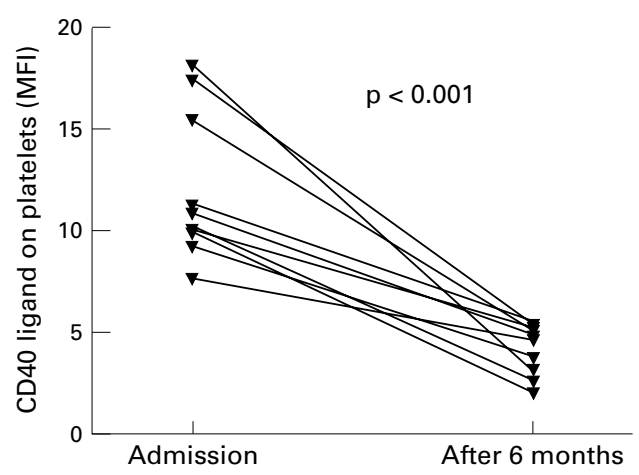

Figure 2 CD4OL/CD154 expression on platelets from 10 patients with unstable angina at admission and at six months follow up $(p<0.001)$. Symbols represent individual measurements (mean fluorescence intensity $((M F I))$.

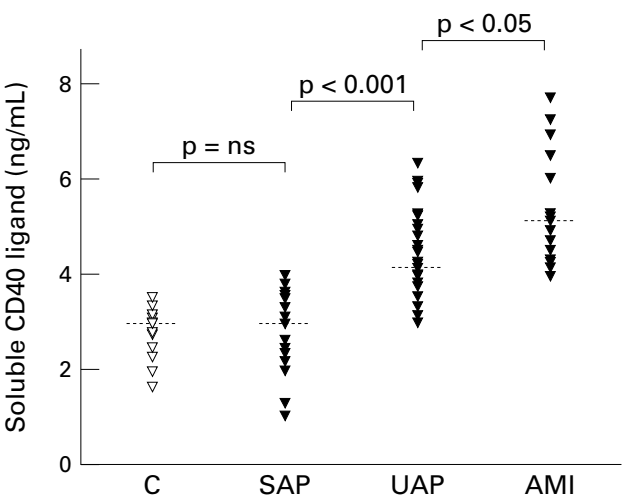

Figure 3 Serum concentrations of soluble CD40L/CD154 from 12 controls $(C), 15$ patients with stable (SAP), 25 patients with unstable angina pectoris (UAP), and 15 patients with acute myocardial infarction (AMI). Symbols represent individual measurements $(\mathrm{ng} / \mathrm{ml})$, the dotted line the mean value of each group (ns, non significant).

(4.2 (0.7) v 5.2 (1.1) ng/ml, p < 0.001), compared with the other study groups (controls $3.0(0.5) v$ stable angina $2.9(1.0) \mathrm{ng} / \mathrm{ml}$, NS). Further analysis showed an inverse correlation between sCD40L/CD154 and surface CD40/CD154 in the infarction group (correlation coefficient $R=-0.3, \mathrm{p}<0.05$ ), but not in unstable angina $(R=-0.15, \mathrm{p}=0.1)$. At the six months follow up, serum concentrations from $\mathrm{sCD} 40 \mathrm{~L} / \mathrm{CD} 154$ in the patients with acute coronary syndromes had reached the range of the control group.

There was a significant increase in $\mathrm{P}$-selectin/CD62P on platelets in patients with stable angina (16.4 (5) MFI), unstable angina (26.9 (11.1) MFI), and myocardial infarction (26.3 (8.4) MFI) compared with the controls (9.4 (2.6); controls $v$ stable angina, unstable angina, or infarction, $\mathrm{p}<0.001$; stable angina $v$ unstable angina or infarction, $\mathrm{p}<0.01$; unstable angina $v$ infarction, NS) (fig 4). Correlation analysis showed that CD40L/CD154 was significantly correlated with P-selectin/ CD62P when all groups were combined $(R=0.47, \quad \mathrm{p}=0.0003)$. Subgroup analysis showed a positive correlation between CD40L/ CD154 and P-selectin/CD62P in the infarction group $(R=0.57, \mathrm{p}=0.04)$, but not in unstable angina $(R=0.37, \mathrm{p}=0.07)$.

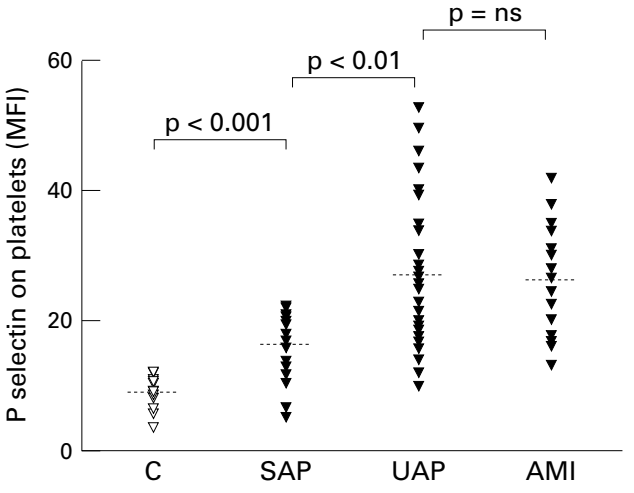

Figure $4 P$-selectin/CD62P expression on platelets in 12 controls (C), 15 patients with stable (SAP), 25 patients with unstable angina (UAP), and 15 patients with acute myocardial infarction (AMI). Symbols represent individual measurements (mean fluorescence intensity ((MFI)), the dotted line the mean value of each group ( $n$, non-significant). 


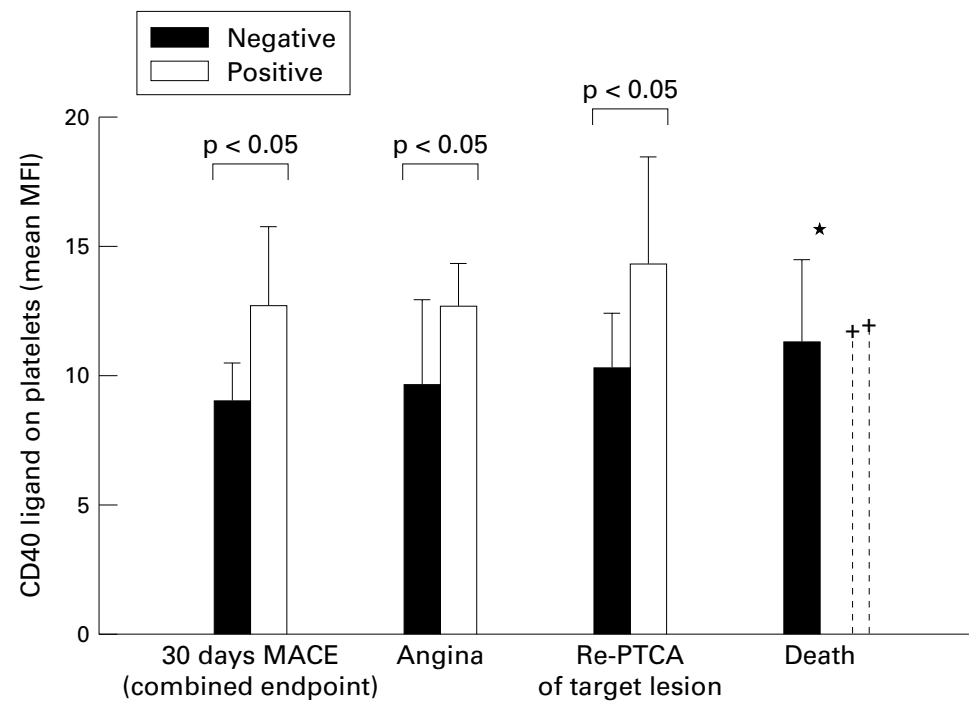

Figure 5 Mean CD40L/CD154 expression on platelets (mean fluorescence intensity $((M F I))$ in relation to major cardiac events within 30 days (30 days $M A C E)$, recurrence of angina, need for repeated PTCA of target lesion, or death. Fourteen of 23 patients with unstable angina fulfilled criteria for 30 days combined end points (white bar), nine patients did not (black bar). Nine patients with unstable angina suffered from recurrence of angina (white bar), whereas 14 patients remained free of symptoms (black bar). Four patients with unstable angina needed repeated PTCA (white bar), the remaining 19 patients with unstable angina did not. Two patients died within 30 days after admission for unstable angina; ${ }^{\star} C D 40 L / C D 154$ on platelets of those who died were within interquartile range of survivors (black bar): both values of those who died (each represented by + ) are placed between the median and third quartile of survivors.

Patients with unstable angina who had major cardiac events within 30 days of their admission (recurrence of angina, need for repeated target vessel PTCA, need for coronary bypass surgery, or death) had a significantly higher mean concentration of surface CD40L/CD154 than those without major cardiac events $(12.7$ (3.1) $v 9.1$ (1.43) MFI, $\mathrm{p}<0.05)$. In addition, among the patients with major cardiac events within 30 days, those with recurrent angina or the need for re-do PTCA had raised CD40L/ CD154 compared with patients who remained symptom-free (recurrence of angina: 12.7 (3.2) v 9.7 (1.6) MFI, p <0.05; re-do PTCA: 14.3 (4.2) v 10.3 (2.1) MFI, p < 0.05) (fig 5). No significant correlations were found between CD40L/CD154 expression on platelets and Braunwald class (IIIB- $T_{\text {negative }}$ or IIIB- $_{\text {positive }}$ ), troponin I concentrations, or ejection fraction. Furthermore, CD40L/CD154 expression on platelets did not correlate with coronary lesion morphology (CD40L/CD154 on platelets in lesion type A $v \mathrm{~B}$, type A $v$ C, or type B $v$ C, all NS) or with TIMI flow grade (CD40L/CD154 on platelets among TIMI flow grades 0, I, II, and III, all NS).

\section{Discussion}

Our study shows an upregulation of CD40L/ CD154 expression on platelets in acute coronary syndromes. Patients with unstable angina and to a lesser extent those with myocardial infarction had significantly higher levels of expression of CD40L/CD154 on their platelets than patients with stable angina or controls. After the acute phase of acute coronary syndromes, at a six months follow up the levels of expression of CD40L/CD154 on platelets were in the range of the controls. In addition, an established marker of platelet activationmembrane bound P-selectin/CD62P - was significantly raised in patients with acute coronary syndromes, and to a lesser extent in those with stable angina compared with controls. Patients with acute coronary syndromes also had raised serum concentrations of sCD40L/CD154. Finally, patients with unstable angina who required re-do PTCA or who had recurrence of angina had increased surface CD40L/CD154 compared with the remainder of the study group.

Because the CD40-CD40L/CD154 interaction promotes plaque destabilisation and the formation of intravascular thrombus, our findings of raised CD40L/CD154 in patients with acute coronary syndromes may have important pathophysiological consequences - upregulated CD40L/CD154 on platelets in patients with unstable angina and myocardial infarction may act as a major contributor to the known local and systemic inflammatory process in acute coronary syndromes. In this context, Mach and colleagues showed enhanced expression of matrix degrading proteinases by macrophages in the shoulder region of atherosclerotic plaques through CD40 signalling, and proposed this as an essential trigger mechanism in acute coronary syndromes. ${ }^{19}$ In addition, Slupsky and associates showed that CD40L/CD154 on activated platelets initiates a procoagulant milieu by inducing tissue factor expression on endothelial cells, like TNF $\alpha .^{22}$

Until now, the finding of enhanced expression of CD40L/CD154 in patients with acute coronary syndromes has been restricted to $\mathrm{CD}^{+}$and $\mathrm{CD} 8^{+} \mathrm{T}$ cells. ${ }^{27} \mathrm{CD} 4^{+} \mathrm{T}$ cells in particular, which typically localise in the shoulder region of advanced human atheromata, express a high percentage of CD40L/CD154 in the peripheral blood of patients with unstable angina. However, as about $10^{12}$ platelets in the circulation carry CD40L/CD154 molecules, significantly enhanced concentrations of CD40L/CD154 on platelets-as shown in this study-may represent an at least equally important activator of endothelial cells, monocytes, fibroblasts, and smooth muscle cells.

Surprisingly, our study shows a discrepancy in the amount of surface CD40L/CD154 on platelets between patients with unstable angina and those with acute myocardial infarction. As patients with myocardial infarction had higher concentrations of soluble CD40L/CD154 but a significantly lower expression of CD40L/ CD154 on platelets compared with patients with unstable angina, it is possible that the patients with myocardial infarction have increased hydrolysis of surface CD40L/CD154. In patients with unstable angina, Aukrust and colleagues recently showed in vitro that there was increased spontaneous release of sCD40L/ CD154 from platelets and a decreased concentration of intracellular sCD40L/CD154; these features were not present in patients with stable angina or controls. ${ }^{27}$ Such an upregulation of the sCD40L/CD154 release mechanism from platelets may be yet further enhanced in patients with acute myocardial infarction, leading to the observed inverse correlation between 
sCD40L/CD154 and surface CD40L/CD154. $\mathrm{Up}$ to now, data on the regulation of CD40L/ CD154 on platelets have not been available, though in the case of CD40L/CD154 on T cells, cleavage of surface CD40L/CD154 by a matrix metalloproteinase has been demonstrated. ${ }^{12}$ Future studies need to determine the exact regulation mechanism of surface CD40L/CD154 on platelets, and the nature of its soluble and biologically active form. ${ }^{32}$

The role of CD40L/CD154 in triggering acute coronary syndromes needs to be clarified further. It can be argued that raised concentration of CD40L/CD154 on platelets are a result rather than a cause of acute coronary syndromes, an argument that cannot be excluded by the data from this study. Nevertheless, if this were the case-and if some other primary event led to the observed increase in concentrations of CD40L/CD154 on platelets-this secondary phenomenon could still induce further activation of the endothelium or monocytes and aggravate the proinflammatory state that finally leads to plaque destabilisation. Thus raised CD40L/CD154 may be both a cause and a consequence of plaque activation.

Beyond this, higher CD40L/CD154 concentrations on admission indicate a complicated 30 days course in hospital, as shown in our study. In view of the proinflammatory role of the CD40 system, this observation is in keeping with previous studies indicating a higher risk of coronary events in patients with increased concentrations of proinflammatory cytokines. ${ }^{33}$

Clinical trials are currently being carried out in patients with systemic lupus erythematosus, using anti-CD40L/CD154 antibodies to downregulate the CD40-CD40L/CD154 interaction. Hence, blocking the CD40-CD40L/ CD154 interaction may become a new therapeutic approach to the treatment of acute coronary syndromes.

\section{LIMITATIONS}

A possible limitation of our study is the platelet-rich plasma method used for preparing platelets before FACS analysis. However, the blood samples taken in this study were collected in an identical fashion from the controls and from the patients with stable angina, unstable angina, and acute myocardial infarction. If our method caused immediate upregulation of surface CD40L/CD154, then the platelets from control donors or patients with stable angina treated the same way would also be expected to contain raised concentrations of surface CD40L/CD154. Instead, platelets from patients with acute coronary syndromes had consistently higher concentrations than platelets from control donors or patients with stable angina. In addition, probe analysis using the blood fixation method has confirmed the observed enhancement of CD40L/CD154 expression on platelets in patients with acute coronary syndromes. ${ }^{34}$ Finally, although we found an association between admission concentrations of CD40L/CD154 on platelets and the clinical outcome, the intention of this study was to investigate whether or not acute coronary syndromes are accompanied by upregulation of surface CD40L/CD154 on platelets; it was not designed or powered to assess the association between surface CD40L/CD154, surface P-selectin/CD62P, or sCD40L/CD154 and clinical end points.

\section{CONCLUSIONS}

The potential importance of our finding of upregulation both of the CD40L/CD154 molecule on platelets, and of the soluble form sCD40L/CD154, in acute coronary syndromes is that this molecule-which has previously been shown to induce a cascade of inflammation by interacting with vascular cells that constitutively display CD40-may represent an essential trigger mechanism in the acute coronary syndromes.

1 Ross R. Atherosclerosis - an inflammatory disease. $N$ Engl f Med 1999;340:115-26.

2 Biasucci LM, Vitelli A, Liuzzo G, et al. Elevated levels of interleukin-6 in unstable angina. Circulation 1996;94:8747.

3 Ikeda H, Takajo Y, Ichiki K, et al. Increased soluble form of P-selectin in patients with unstable angina. Circulation 1995;92:1693-6.

4 Mazzone A, De Servi S, Ricevuti G, et al. Increased expression of neutrophil and monocyte adhesion molecules in unstable coronary artery disease. Circulation 1993;88:35863.

5 Neri Serneri GG, Prisco D, Martini F, et al. Acute T-cell activation is detectable in unstable angina. Circulation 1997;95:1806-12.

6 Kato K, Santana-Sahagun E, Rassenti LZ, et al. The soluble CD40 ligand sCD154 in systemic lupus erythematosus. $\mathcal{F}$ CD40 ligand SCD154 in system $1999 ; 104: 947-55$.

7 Mach F, Schonbeck U, Libby P. CD40 signaling in vascular cells: a key role in atherosclerosis? Atherosclerosis 1998; 137(suppl):S89-95.

8 Mach F, Schonbeck U, Sukhova GK, et al. Reduction of atherosclerosis in mice by inhibition of CD40 signalling. Nature 1998;394:200-3.

9 Schonbeck U, Sukhova GK, Shimizu K, et al. Inhibition of CD40 signaling limits evolution of established atherosclerosis in mice. Proc Natl Acad Sci USA 2000;97:7458-63.

10 Lutgens E, Gorelik L, Daemen MJ, et al. Requirement for CD154 in the progression of atherosclerosis. Nat Med 1999;5:1313-16.

11 Lutgens E, Cleutjens KB, Heeneman S, et al. Both early and delayed anti-CD40L antibody treatment induces a stable plaque phenotype. Proc Natl Acad Sci USA 2000;97:7464-9.

plaque phenotype. Proc Natl Acad $S c i U S A$ ropos
12 Phipps RP. Atherosclerosis: the emerging role of inflammation and the CD40-CD40 ligand system. Proc Natl Acad Sci tion and the CD40-CD $2000 ; 97: 6930-2$.

13 Lederman S, Yellin MJ, Krichevsky A, et al. Identification of a novel surface protein on activated $\mathrm{CD} 4+\mathrm{T}$ cells that nduces contact-dependent B cell differentiation (help). f Exp Med 1992;175:1091-101.

14 Gauchat JF, Henchoz S, Mazzei G, et al. Induction of human IgE synthesis in B cells by mast cells and basophils. Nature 1993;365:340-3.

15 Mach F, Schonbeck U, Sukhova GK, et al. Functional CD40 ligand is expressed on human vascular endothelial cells, smooth muscle cells, and macrophages: implications for CD40-CD 40 ligand signaling in atherosclerosis. Proc Natl Acad Sci USA 1997;94:1931-6.

16 Alderson MR, Armitage RJ, Tough TW, et al. CD40 expression by human monocytes: regulation by cytokines and activation of monocytes by the ligand for CD40. F Exp Med 1993;178:669-74.

17 Karmann K, Hughes CC, Schechner J, et al. CD40 on human endothelial cells: inducibility by cytokines and human endothelial cells: inducibility by cytokines and
functional regulation of adhesion molecule expression. Proc functional regulation of adhesion mol

18 Hollenbaugh D, Mischel-Petty N, Edwards CP, et al. Expression of functional CD40 by vascular endothelial cells. F Exp Med 1995;182:33-40.

19 Mach F, Schonbeck U, Bonnefoy JY, et al. Activation of monocyte/macrophage functions related to acute atheroma complication by ligation of CD40: induction of collagenase, stromelysin, and tissue factor. Circulation 1997;96:396-9.

20 Thienel U, Loike J, Yellin MJ. CD154 (CD40L) induces human endothelial cell chemokine production and migration of leukocyte subsets. Cell Immunol 1999; 198:87-95.

21 Miller DL, Yaron R, Yellin MJ. CD40L-CD40 interactions regulate endothelial cell surface tissue factor and thrombomodulin expression. F Leukoc Biol 1998;63:373-9.

22 Slupsky JR, Kalbas M, Willuweit A, et al. Activated platelets induce tissue factor expression on human umbilical vein endothelial cells by ligation of CD40. Thromb Haemost 1998;80:1008-14. 
23 Henn V, Slupsky JR, Grafe M, et al. CD40 ligand on activated platelets triggers an inflammatory reaction of activated platelets triggers an inflammator
endothelial cells. Nature 1998;391:591-4.

24 Fitzgerald DJ, Roy L, Catella F, et al. Platelet activation in unstable coronary disease. N Engl f Med 1986;315:983-9.

25 Ott I, Neumann FJ, Gawaz M, et al. Increased neutrophilplatelet adhesion in patients with unstable angina. Circulation 1996;94:1239-46.

26 Lee Y, Lee WH, Lee SC, et al. CD40L activation in circulating platelets in patients with acute coronary syndrome. ing platelets in patients
Cardiology 1999;92:11-16.

27 Aukrust P, Muller F, Ueland T, et al. Enhanced levels of soluble and membrane-bound CD40 ligand in patients with unstable angina. Possible reflection of T lymphocyte and platelet involvement in the pathogenesis of acute coronary syndromes. Circulation 1999;100:614-20.

28 Hamm CW, Braunwald E. A classification of unstable angina revisited. Circulation 2000;102:118-22.

29 Gawaz M, Ott I, Reininger AJ, et al. Agglutination of isolated platelet membranes. Arterioscler Thromb Vasc Biol 1996;16: p21-7.
30 Ryan TJ, Faxon DP, Gunnar RM, et al. Guidelines for percutaneous transluminal coronary angioplasty. A report of cutaneous transluminal coronary angioplasty. A report of the American College of Cardiology/American Heart Association task force on assessment of diagnostic and therapeutic cardiovascular procedures (subcommittee on percutaneous transluminal coronary angioplasty). Circulation 1988;78:486-502

31 TIMI Study Group. The Thrombolysis in Myocardial Infarction (TIMI) trial. Phase I findings. $N$ Engl $\mathcal{F}$ Med 1985;312:932-6.

32 Mazzei GJ, Edgerton MD, Losberger C, et al. Recombinant soluble trimeric CD40 ligand is biologically active. $\mathcal{F}$ Biol Chem 1995;270:7025-8.

33 Biasucci LM, Liuzzo G, Fantuzzi G, et al. Increasing levels of interleukin (IL)-1Ra and IL-6 during the first 2 days of hospitalization in unstable angina are associated with in 1999;99:2079-84.

34 Shattil SJ, Cunningham M, Hoxie JA. Detection of activated platelets in whole blood using activation-dependent monoplatelets in whole blood using activation-dependent mono-
clonal antibodies and flow cytometry. Blood 1987;70: 307-15.

\section{IMAGES IN CARDIOLOGY}

\section{Artefactual coronary artery lesions caused by effect of guidewire on tortuous coronary arteries: angiographic appearances during right coronary angioplasty}

The angiographic appearances of complications during coronary angioplasty such as coronary artery dissection, spasm or thrombus formation are well recognised. These situations require specific corrective measures such as stenting, intracoronary nitrates or infusion of glycoprotein IIb/IIIa inhibitors. However, not all coronary artery abnormalities seen during coronary angioplasty are true lesions that require treatment. We report a case of lesions appearing in the proximal vessel during right coronary angioplasty that were artefactual.

Panel A below shows the diagnostic angiogram with the original stenosis in distal right coronary artery arrowed. In panel $\mathrm{B}$, this stenosis has been successfully stented, but apparent severe stenoses (bold arrow) have appeared at the site of vessel tortuosity in the proximal segment of the "shepherd's crook" right coronary artery, with the guidewire in situ. In panel $\mathrm{C}$, the guidewire has been withdrawn allowing the right coronary artery to assume its natural curvature and the lesions have disappeared.

The apparent lesions were the result of the guidewire straightening out tortuous segments of the artery and causing vessel wall invagination, also described as the "accordion effect". These angiographic appearances must be recognised to avoid unnecessary stenting of arterial segments that are not diseased. The guidewire should be withdrawn until the floppy tip lies within the suspected segment to enable the vessel to resume its original shape, so that the diagnosis of an artefactual lesion can be confirmed without losing guidewire position (panel C). The right coronary artery is thought to be particularly susceptible to this phenomenon because the artery lies relatively unsupported by surrounding tissue in the atrioventricular groove.

$\mathrm{T}$ W $\mathrm{KOH}$

P KELLY

A D TIMMIS tatkoh@hotmail.com

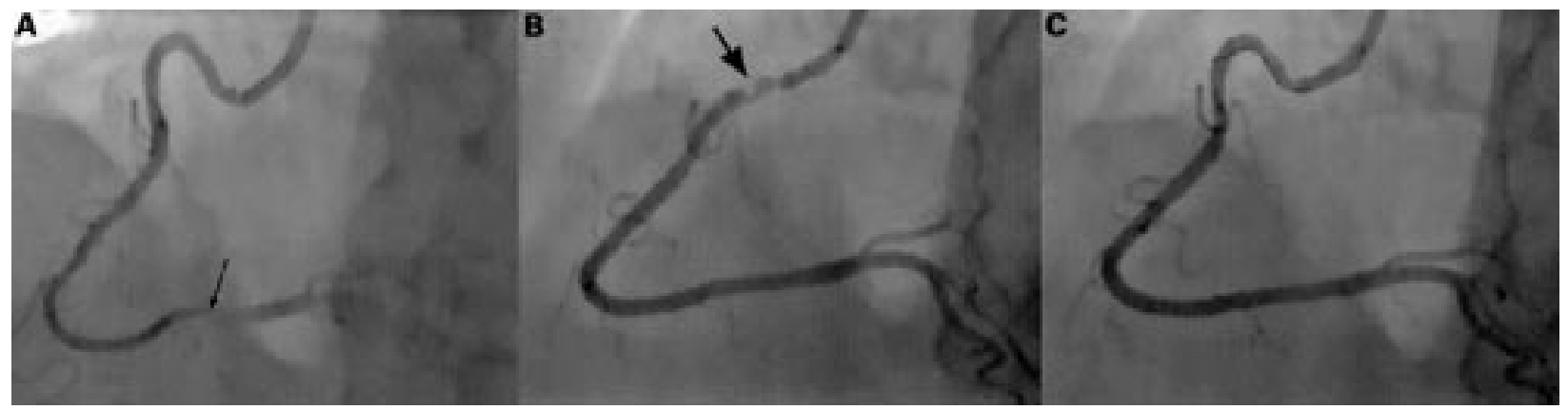

